\title{
Cocaina e insufficienza renale acuta
}

\author{
M. Terribile, D. Paracuollo, F. D’Anna, R. Genualdo, R. Rossano, M. Terribile, \\ M. Paracuollo, M. Capuano
}

Unità Operativa Complessa di Nefrologia e Dialisi, Ospedale dei Pellegrini, ASL Napoli 1, Napoli

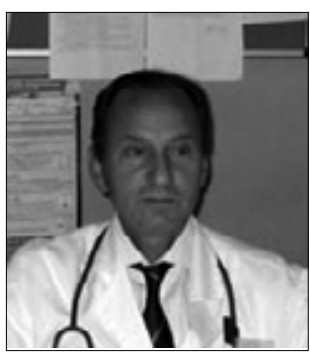

Maurizio Terribile

\section{Introduzione}

Il consumo di cocaina $(C)$ è sempre più comune. In base ad analisi dei capelli, si stima che il 6\% della popolazione degli USA ne faccia uso (1). La $\mathrm{C}$ è una sostanza i cui effetti possono riguardare vari sistemi ed apparati in relazione sia alla sua tossicità diretta che alle azioni mediate dall'interferenza sul metabolismo della dopamina e delle catecolamine. Infatti la C esalta gli effetti della stimolazione simpatica inibendo il re-uptake delle catecolamine nelle terminazioni nervose. In tal modo si prolungano e si esaltano gli effetti del sistema simpatico.

Può essere pertanto coinvolto: a) l'apparato cardiovascolare (2) con cardiopatia ischemica, infarto del miocardio, aritmie, miocarditi, ipertensione arteriosa, dissezione aortica; b) neurologico (3) con convulsioni, ictus, agitazione, atrofia cerebrale; c) polmonare 4) con asma, broncopolmoniti, emorragia ed infarto polmonare, embolia polmonare, crack lung; d) gastrointestinale (5) con ischemie intestinali, infarti mesenterici, perforazioni intestinali, coliti; e) muscolo scheletrico con rabdomiolisi.

Il rene, in relazione al suo primario ruolo di organo emuntorio, rappresenta uno dei principali bersagli dell'azione tossica della $\mathrm{C}$; contribuiscono indubbiamente gli alti livelli di concentrazione raggiunti dalla sostanza e dai suoi metaboliti nel parenchima renale.

Nella Tabella I sono riportati gli effetti della C sul rene.

TABELLA I - EFFETTI SUL RENE DA ABUSO DI COCAINA (6)

Insufficienza renale acuta per:

Rabdomiolisi

Ipertensione maligna

Nefrite interstiziale

GN con Ab anti-membrana basale

Microangiopatia trombotica

Alterazioni del sistema acido-base

Alterazioni del sistema idro-elettrolitico

Infarto renale

Infezione vie urinarie

Insufficienza renale cronica per:

Ipertensione arteriosa mal controllata

Ruolo della C nella progressione della IRC

Abbiamo riscontrato 4 casi clinici di insufficienza renale acuta (IRA) da C negli ultimi 18 mesi. Nella Tabella II sono riportati i dati clinici e laboratoristici iniziali dei pazienti da noi studiati.

TABELLA II - DATI CLINICI E LABORATORISTICI INIZIALI DEI 4 PAZIENTI CON IRA DA C

\begin{tabular}{|l|c|c|c|c|c|c|c|c|c|}
\hline & $\begin{array}{c}\text { Età } \\
\text { (anni) }\end{array}$ & Sesso & Via di assunzione & $\begin{array}{c}\text { Tempo } \\
\text { intercorso ns } \\
\text { osservazione } \\
\text { (giorni) }\end{array}$ & $\begin{array}{c}\text { Pressione } \\
\text { arteriosa } \\
(\mathrm{mmHg})\end{array}$ & $\begin{array}{c}\text { Creat. } \\
(\mathrm{mg} \%)\end{array}$ & $\begin{array}{c}\text { Mioglobinemia } \\
\text { (ng/mL) }\end{array}$ & $\begin{array}{c}\text { CPK } \\
\text { (U/L) }\end{array}$ & $\begin{array}{c}\text { GOT } \\
\text { (U/L) }\end{array}$ \\
\hline & & & & & & & & & \\
\hline Caso 1 & 43 & $\mathrm{M}$ & Nasale & 3 & $160 / 100$ & 12,0 & $>3000$ & 26800 & 533 \\
\hline Caso 2 & 25 & $\mathrm{M}$ & Nasale & 2 & $150 / 90$ & 10,2 & $>3000$ & 11300 & 430 \\
\hline Caso 3 & 19 & $\mathrm{M}$ & Intestinale & 2 & $160 / 80$ & 12,9 & $>2000$ & 15800 & 551 \\
\hline Caso 4 & 39 & $\mathrm{~F}$ & Nasale & 1 & $70 / 50$ & 5,2 & $>5000$ & 12700 & 644 \\
\hline
\end{tabular}




\section{Caso 1}

Paziente D. M. di 43 anni, HCV positivo, tossicodipendente. In seguito ad overdose di cocaina, assunta per via inalatoria (circa 10 grammi), presenta mialgie diffuse, oliguria, urine color marrone. Dopo 3 giorni effettua esami laboratoristici che evidenziano IRA (azotemia $218 \mathrm{mg} / 100 \mathrm{~mL}$; creatinina $12 \mathrm{mg} / 100 \mathrm{~mL}$; Na $131 \mathrm{mEq} / \mathrm{L} ; \mathrm{K} 6,4 \mathrm{mEq} / \mathrm{L}$ ). Si ricovera pertanto presso la nostra Unità Operativa dove si rileva altresì rabdomiolisi $(\mathrm{R})$ con mioglobinemia $>3000 \mathrm{ng} / \mathrm{mL}$; GOT 533 U/L; GPT 382 U/L; LDH 3197 U/L; CPK 26800 U/L; mioglobinuria. È presente positività per i metabolici della $\mathrm{C}$. Si rileva altresì moderata ipertensione arteriosa (P.A. 160/100) e grave acidosi metabolica. Il paziente effettua 5 sedute emodialitiche per poi presentare una ripresa della diuresi con un miglioramento della funzionalità renale. Dopo 15 giorni dall'evento tossico il paziente viene dimesso con valori di creatininemia di $2,5 \mathrm{mg} \%$ che si normalizzeranno nell'arco di 1 mese.

\section{Caso 2}

Paziente R. F. di 25 anni, con normofunzione renale, in seguito ad overdose di cocaina (circa 14 grammi inalati) sviluppa perdita di coscienza, rimanendo nella stessa posizione per lungo tempo. Trasportato in Ospedale, si evidenzia grave $\mathrm{R}$ (mioglobinemia > $3.000 \mathrm{ng} / \mathrm{mL}$; CPK $11.300 \mathrm{U} / \mathrm{L} ; \mathrm{LDH}$ 1220 U/L; GOT 430 U/L; mioglobinuria). Si evidenzia positività per i metabolici della $\mathrm{C}$. Nei giorni successivi sviluppa oligoanuria ed IRA (azotemia $230 \mathrm{mg} \%$, creatininemia 10,2 $\mathrm{mg} \%$ ) con grave acidosi metabolica. È pertanto trasferito presso la nostra Unità Operativa, ove effettua 7 trattamenti dialitici. Successivamente presenta però un peggioramento clinico, con crampi e dolori muscolari diffusi; si sviluppa una sindrome compartimentale con risalita contestuale di mioglobinemia, CPK, LDH e transaminasi. Presenta altresì grave iperpotassiemia $(7,5 \mathrm{mEq} / \mathrm{L})$ e marcata acidosi metabolica. Viene emodializzato in modo intensivo con ritmo quotidiano per 7 giorni, e la programmata fasciotomia non viene più effettuata per una lenta e progressiva ripresa clinica e della funzionalità renale. Nella fase poliurica necessita ancora di trattamento emodialitico per ipercalcemia $(13,1 \mathrm{mg} \%)$ ed iperfosforemia $(10,6 \mathrm{mg} \%)$.

E poi dimesso dopo circa 30 giorni con creatininemia di 2 $\mathrm{mg} \%$ e Clearance creatinina di $40 \mathrm{ml} / \mathrm{min}$.

\section{Caso 3}

Paziente C.L. di 19 anni, tossicodipendente e dedito allo spaccio di stupefacenti. Per trasporto illecito, ingerisce 5 ovuli di cocaina ed eroina, ma per rottura accidentale di 1 ovulo presenta intossicazione acuta da $\mathrm{C}$ ed eroina. Svi- luppa perdita di coscienza e permane in immobilizzazione prolungata in postura inusuale, con compressione muscolare e voluminoso ematoma all'emilato destro. Dopo 24 ore viene trasportato all'Ospedale più vicino dove si evidenzia $R$ (mioglobinemia > $2000 \mathrm{ng} / \mathrm{mL}$; CPK $15800 \mathrm{U} / \mathrm{L}$; LDH 8260 U/L; GOT $551 \mathrm{U} / \mathrm{L}$; mioglobinuria) ed IRA oligoanurica (azotemia $255 \mathrm{mg} \%$; creatininemia 12,9 mg\%; Na $130 \mathrm{mEq} / \mathrm{L}$ e K 7,5 mEq/L; uricemia $15 \mathrm{mg} \%$ ). E altresì presente marcata acidosi metabolica (pH 7,25; $\mathrm{HCO} 316 \mathrm{mEq} / \mathrm{L} ; \mathrm{pCO} 2$ $36 \mathrm{mmHg}$ ). Gli esami tossicologici evidenziano positività per i metabolici della $\mathrm{C}$ ed eroina. Viene trasferito dopo 24 ore presso la nostra Unità Operativa e sottoposto ad immediato trattamento emodialitico. Si manifesta altresì una coagulazione intravasale disseminata (CID) con prolungamento del PT e PTT, trombocitopenia e ipofibrinogenemia; è necessario il ricovero in Terapia Intensiva ove viene sottoposto a terapia con AT III ed eparina. Sono effettuati 8 trattamenti emodialitici con una ripresa funzionale fino ad una normalizzazione dei parametri renali. È poi dimesso dopo 25 giorni di ricovero.

\section{Caso 4}

Paziente C.V. di 39 anni, in normofunzione renale. Fa uso abituale di cocaina da circa 4 anni. In seguito ad overdose da C (circa 15 grammi per via intranasale) il paziente presenta obnubilamento del sensorio. Dopo circa 24 ore è accompagnato al Pronto Soccorso del nostro Ospedale e ricoverato in Terapia Intensiva essendo le condizioni generali molto critiche (P max $70 \mathrm{mmHg}$; $\mathrm{pH} 7,12$; HCO3 $7 \mathrm{mEq} / \mathrm{L} ; \quad$ pCO2 $44 \mathrm{mmHg}$ ). Il paziente presenta anuria da circa 12 ore ed IRA (creatininemia $5,2 \mathrm{mg} \%$; $\mathrm{K}$ $7,4 \mathrm{mEq} / \mathrm{L}$; Na $140 \mathrm{mEq} / \mathrm{L}$; uricemia $10,5 \mathrm{mg} \%$; mioglobinemia > 5000 ng/mL; CPK 12700 U/L; LDH 7800 U/L; GOT $644 \mathrm{U} / \mathrm{L}$; mioglobinuria). Viene trattato con CAVHDF per circa 50 ore. Al terzo giorno sospende il trattamento continuo per la ripresa della diuresi e per un notevole miglioramento dei parametri laboratoristici. È poi dimesso dopo 10 giorni con creatininemia $1,9 \mathrm{mg} \%$. Successivamente non si presenta ai nostri controlli clinici programmati.

\section{Discussione}

La principale causa di insufficienza renale acuta conseguente all'uso di cocaina è la R (7). L'associazione C-R è ampiamente riportata in letteratura $(8,9)$. Anche in tutti i 4 nostri casi clinici si è evidenziata tale associazione. I meccanismi patogenetici alla base della $\mathrm{R}$ da $\mathrm{C}$ sono molteplici. É stato dimostrato: a) un effetto tossico diretto della $\mathrm{C}$ sulla muscolatura scheletrica $(10)$; b) un 
effetto indiretto di ipossia e ischemia conseguente alla vasocostrizione prolungata (11); c) un danno da compressione muscolare secondaria a perdita di coscienza. La $\mathrm{R}$ potrebbe essere inoltre causata dalla presenza di sostanze contaminanti quali la stricnina, la fenciclidina, l'arsenico e le anfetamine, sostanze di cui è nota la capacità di causare convulsioni o R (12).

Caratteristica della $\mathrm{R}$ è la mioglobinuria, da noi sempre riscontrata. La precipitazione intratubulare di mioglobina con conseguente ostruzione è la principale causa del danno renale.

La marcata acidosi metabolica, da noi rilevata in tutti i pazienti, ha molteplici cause: la liberazione di acido lattico dai muscoli ischemici o ipoperfusi, la liberazione di amminoacidi solforati dai muscoli in necrosi, la liberazione di acido urico dalla lisi cellulare, la perdita di bicarbonati che si legano al calcio nei muscoli danneggiati, ed infine la stessa acidosi uremica.

La grave iperpotassiemia, da noi sempre riscontrata, è ascrivibile non solo alla acidosi metabolica ma anche alla massiva liberazione di potassio dalle cellule muscolari lisate, e all'IRA oligo-anurica.

La ipercalcemia, rilevata nel Caso 2 durante la fase poliurica, è conseguente alla rapida mobilizzazione di calcio da muscoli danneggiati.

La concomitanza di iperfosforemia, legata alle stesse cause, ha richiesto un rapido intervento con emodialisi per evitare la precipitazione metastatica di fosfato di calcio nei vari tessuti, con un possibile peggioramento della funzione renale. La CID, riscontrata nel Caso 3, dovuta allimmissione in circolo di attivatori della protrombina conseguente alla $\mathrm{R}$, è un evento non raro e potenzialmente letale. La pronta terapia attuata ha consentito di evitare un quadro di CID acuta e progressiva, permettendoci un buon controllo sugli eventi coagulativi.

La Sindrome da compartimentazione, particolarmente evidente nel Caso 2, ha generato un aumento di pressione tessutale sui muscoli, che ha riacutizzato la lisi, ma non è stata tale da provocare danno nervoso e paralisi da edema compressivo.

Da rilevare come in tutti i casi sia stato necessario far ricorso alla terapia dialitica. Infatti il "late referral" alla nostra struttura nefrologica non ha consentito di istituire terapie farmacologiche, con adeguata idratazione mediante bicarbonato di sodio, che avrebbero probabilmente potuto prevenire l'IRA. Non abbiamo infine mai riscontrato gravi ipertensioni arteriose e pensiamo che tale mancato rilievo sia anch'esso da correlarsi al "late referral" presso la nostra struttura. Infatti la grave ipertensione arteriosa è molto spesso un momento iniziale della intossicazione da $\mathrm{C}$, in quanto nelle giornate successive i meccanismi di compenso tendono a limitare il fenomeno.
In conclusione, poiché l'uso di $\mathrm{C}$ ha raggiunto proporzioni epidemiche, è sicuramente auspicabile l'esecuzione routinaria di uno screening tossicologico nelle persone giovani afferenti alle strutture di emergenza con assenza o minimo rischio cardiovascolare, ma in preda a quadri acuti di compromissione renale e/o cardiaca.

\section{Riassunto}

Il consumo di cocaina ha raggiunto proporzioni epidemiche. Le complicanze renali correlate al suo abuso sono sempre più frequentemente evidenziate. Descriviamo 4 casi clinici di insufficienza renale acuta da overdose di cocaina da noi riscontrati negli ultimi 18 mesi. La insufficienza renale era in tutti i casi correlata a rabdomiolisi, che è il meccanismo patogenetico più comune. A causa del "late referral" alla nostra struttura, non è stata effettuata una pronta ed efficace terapia medica ed è stato necessario in tutti i casi istituire trattamento emodialitico prima della riprese funzionale dell'organo.

Parole chiave: Insufficienza renale acuta, Cocaina, Rabdomiolisi

\section{TEST di VERIFICA - 8}

1) Qual è la causa più comune di insufficienza renale acuta da cocaina?
a) Infarto renale
b) Rabdomiolisi
c) Ipertensione maligno
d) Trombosi vasi renali
e) Disidratazione

2) Qual è il trattamento migliore per prevenire l'insufficienza renale acuta da rabdomiolisi?
a) Terapia diuretica
b) Allopurinolo
c) Bicarbonato
d) Fisiologica
e) Terapia cortisonica

\section{3) L'acidosi metabolica nella IRA da cocaina è riconducibile a}
a) Acidosi uremica
b) Aumento di acido lattico
c) Aumento di acido urico
d) Tutte le precedenti

Le risposte corrette alle domande sono a pag. 24 
Indirizzo degli Autori:

Maurizio Terribile, MD

Unità Operativa di Nefrologia e Dialisi

Ospedale dei Pellegrini- ASL Napoli 1 Centro

Via Portamedina alla Pignasecca 41

80134 Napoli

maurizio.terribile@fastwebnet.it

\section{Bibliografia}

1. Kidwell DA, Blanco MA. Cocaine detection in a university population by hair analysis and skin swab testing. Forensic Sci Int 1997; 84: 75-86.

2. Hollander J, Hoffman R. Cocaine-induced myocardial infarction, an analysis and review of the literature. J Emerg Med 1992; 10: 169-77.

3. Daras M, Tuchman A. Central nervous system infarction related to cocaine use. Stroke 1991; 22: 1320-25.

4. Itkonen J, Schnoll S. Pulmonary dysfunction in freebase cocaine users. Arch Int Med 1994; 144: 2195-97.

5. Nolbandian H, Sheth N. Intestinal ischemia caused by co- caine ingestion. Surgery 1995; 97: 374-76.

6. Nzerue CM, Hewan-Lowe K, Riley LJ. Cocaine and the kidney: a synthesis of pathophysiologic and clinical perspectives. Am J Kidney Dis 2000; 35(5): 783-95.

7. Roth D, Alarcon FJ, Bourgiognie JJ, et al. Acute rhabdomyolysis associated with cocaine intoxication. $\mathrm{N}$ Engl J Med 1988; 319: 673-7.

8. Horowitz BZ, Panacek EA, Jouriles NJ. Severe rhabdomyolysis with renal failure after intranasal cocaine use. J Emerg Med 1997; 15(6): 833-7.

9. Counselman FL, McLaughlin EW, Kardon EM, Bhambhani-Bhavnani AS. Creatine phosphokinase elevation in patients presenting to the emergency department with cocaine-related complaints. Am J Emerg Med 1997;15(3): 221-3.

10. Pagala M, Amaladevi B, Azad D, Pagala S, Herzlich B, Namba T, Grob D. Effect of cocaine on leakage of creatine kinase from isolated fast and slow muscles of rat. Life Sci 1993; 52(8): 751-6.

11. Welch RD, Todd K, Krause GS. Incidence of cocaineassociated rhabdomyolysis. Ann Emerg Med 1991; 20(2):154-7.

12. Crowe AV, Howse M, Bell GM, and Henry JA. Substance abuse and the kidney. Q J Med 2000; 93: 147-52. 